\title{
Constraints on Flows in Hořava-Lifshitz Gravity by Classical Solutions
}

\author{
Taekyung Kim $*$ and Yoonbai Kim \\ Department of Physics, BK21 Physics Research Division, and Institute of Basic Science, \\ Sungkyunkwan University, Suwon 440-746, Korea
}

\begin{abstract}
We find exact static stringy solutions of Horava-Lifshitz gravity with the projectability condition but imposing the detailed balance condition near the UV fixed point, and propose a method on constraining the possible pattern of flows in Hořava-Lifshitz gravity by using the obtained classical solutions. In the obtained vacuum solutions, the parameters related to the speed of the graviton and the coefficients of quartic spatial derivative terms lead to intriguing effects: the change of graviton speed yields a surplus angle and the quartic derivatives make the square of effective electric charge negative. The result of a few tests based on the geometries of a cone, an excess cone, a black string, and a charged (black) string seems suggestive. For example, the flow of constant graviton speed and variable Newton's coupling can be favored in the vicinity of an IR fixed point, but the conclusion is indistinct and far from definite yet. Together with the numerous classical solutions, static or time dependent, which have already been found, the accumulated data from various future tests will give some hints in constraining the flow patterns more deterministic.
\end{abstract}

\footnotetext{
*Electronic address: pojawd@skku.edu
}

$\dagger$ Electronic address: yoonbai@skku.edu 


\section{Motivation and Proposal}

Hořava-Lifshitz (HL) gravity of $z=3$ anisotropic scaling,

$$
t \rightarrow \ell^{z} t, \quad x^{i} \rightarrow \ell x^{i}
$$

has been proposed to achieve a theory of quantized gravity, which is believed to be ultraviolet (UV) complete and unitary [1 4]. It is based on the assumption that there exists renormalization group (RG) flow connecting a higher spatial derivative gravity such as the Lifshitz type UV fixed point and general relativity (GR) such as the infrared (IR) fixed point. Though nonexistence of ghost excitation is shown by analyzing the tree-level propagator and it is believed to be power-counting renormalizable due to sixth order spatial derivatives, the HL gravity in its various versions encounters phenomenological inconsistencies, e.g., unwanted dynamical scalar degree and the related strong gravitational coupling [1, 5, 6], and theoretical difficulties in quantization procedure [7]. A newly proposed version of HL gravity is intriguing since an extra local U(1) symmetry to the foliation-preserving diffeomorphism fixes $\lambda$ to be unity and eliminates the scalar graviton [2].

In this infant stage of HL gravity it seems difficult to discuss specifically the RG flows connecting the IR and UV fixed points: Not even a single research including direct computation of $\mathrm{RG}$ in the context of HL gravity has been performed yet. It is not so puzzling since, except for some supersymmetric gauge theories or lower-dimensional field theories, it is difficult even in flat $(1+3)$-dimensional spacetime to find an RG flow possessing both IR and UV fixed points.

A step aside from the issues related to propagating degrees and quantization, there is also an intriguing research direction to find classical configurations by solving equations of motion. In fact, numerous classical solutions have been obtained in diverse directions, among which the large portion consists of time-dependent cosmological solutions [8-10] and static black hole solutions [913. Many of those are given by exact solutions despite complication due to the higher derivative nonlinear equations. These classical configurations contribute obviously to the understanding classical nature of HL gravity. However, different from the case of GR or Newtonian gravity, direct detection of the evidences of HL gravity from the obtained classical configurations seems extremely difficult in both astrophysical observation in large scale and laboratory experiments [12, 14, 15].

A natural question is what can be the usefulness of various classical solutions for the ultimate goal of HL gravity, a quantum gravity. A specific question may be that whether or not the obtained classical solutions, at least some solutions, can be utilized to constrain possible patterns of the flows connecting the IR and UV fixed points which are presumed to be GR and HL gravity based on $z=$ 3 anisotropic scaling, respectively. Although it is speculative, we will try to address this question in this paper. The procedure is given as follows. First, we consider HL gravity involving the square of the Cotton tensor as the unique sixth order spatial derivative term, but the coefficients of lower spatial derivative terms satisfying the foliation-preserving diffeomorphism remain to be arbitrary. Second, we solve the equations of motion in the coordinates compatible with the assigned symmetries and conditions, find classical configurations, in which some of those are given as exact 
solutions, and read the corresponding geometries. Third, we consider a multidimensional map of parameters of the theory, and the obtained solutions are utilized in dividing the regions in the map. There are 10 parameters composed of 8 coefficients in front of the Lagrangian terms and 2 more constants in the classical solutions. The selected classical solutions for application to RG flows are required to possess the following nature:

1. The solution is generic in the context of HL gravity. Here "generic" means that the solution is obtained not under a bizarre metric assumption, not by too many surgeries of different geometries, and not in the presence of unphysical matters, unphysical in the scheme of field theories with Lifshitz type anisotropic scaling.

2. A solution is found in the wide range of parameters.

3. Solution reduces to a physically acceptable solution in the GR limit.

Note that the aforementioned static solutions fulfill the requirements. Fourth, we examine characteristic of each solution (geometry) and classify the corresponding region. The obtained solutions in the realm of HL gravity are categorized as follows in comparison with GR solutions:

(I) The usual solutions which appear as physical solutions in GR and of which the corresponding geometries are observed directly or/and indirectly.

(II) The solutions which are encountered in GR but of which their astrophysical signals seem hardly or unlikely to be detected.

(III) The solutions which are never obtained in GR or cannot be obtained in the physical environment of GR, e.g., unphysical solutions under violation of the positive energy theorem.

When a parameter changes smoothly along the line of RG flow at least in the vicinity of the IR fixed point, a classical GR solution changes to be a classical HL solution in one of the above three categories. If it is category (I), we may call the corresponding parameter region an "allowed zone." If it is category (II), we may call the corresponding parameter region a "disfavored zone." If it is category (III), we may call the corresponding parameter region an "unlikely zone."

In the subsequent sections, we find classical solutions describing some stringy objects and test our proposal. In Sec. 2, without matter, we solve the equations of motion in the PainlevéGullstrand type coordinates compatible with the projectability condition and find exact axially symmetric solutions of which planar geometries describe a cone with deficit angle, an excess cone with surplus angle, and a black string with a Banados-Teitelboim-Zanelli (BTZ) type horizon. In order to obtain the cone and excess cone configurations in GR, depicted by the same metric, negative energy density is required in the energy-momentum tensor side of the Einstein equations. Therefore, the solution is unphysical in the context of GR. In the vicinity of the IR fixed point with vanishing higher spatial derivative terms, these solutions belong to category (III) and the parameter region supporting these configurations may correspond to an unlikely zone. We categorize the black string into (II) and the parameter region of this solution may correspond to a disfavored 
zone. An allowed zone keeps the speed of light propagation unity but the gravitational coupling can be changed. In Sec. 3 we solve the equations of motion in the presence of an electrostatic field from a thin filament of charge and find a charged (black) string solution [with Reissner-Nordström $(\mathrm{RN})$ type horizons]. In this metric the sum of the square of the electric charge and a combination of coefficients in front of two curvature square terms appears so that this square of effective charge is not positive semidefinite. Even in the presence of electrostatic field with positive energy density, there is a static solution in which square of the effective charge is negative. It means that the obtained solution is generic in HL gravity. However, in the context of GR, the energy density given by the square of the electric field is required to be negative in order to get the same metric solution. In classical electrodynamics, this can be interpreted as an attractive Coulomb force between the same charges. Since the former violates the positive energy theorem in GR and the latter is not allowed in the Maxwell theory, the solution may belong to category (III) and the corresponding parameter region may be an unlikely zone. Since the astrophysical objects with a huge amount of excess charge can hardly be formed due to repulsive nature, the black string solution with the charge greater than a critical value may be categorized into (II) and the corresponding parameter region may be a disfavored zone. In Sec. 4 we summarize briefly what we obtained. Then we discuss possible obstacles to jeopardize this speculative proposal and the conditions to put this suggestion forward to a plausible scenario.

\section{Vacuum Solution and Flow for Quadratic Derivative Terms}

In this section we consider HL gravity with the projectability condition but impose the detailed balance condition only in the UV limit. In Sec. 2.1, we look for exact classical static vacuum solutions with axial symmetry, and the obtained configurations can describe the straight stringy objects stretched infinitely along the $z$ axis. In Sec. 2.2, we discuss a possible pattern of the RG flows by applying the obtained solutions to the method proposed in Sec. [1.

The HL gravity action in $(1+3)$ dimensions is given by

$$
S_{\mathrm{HL}}=\int d t d^{3} x \sqrt{g} N\left(\mathcal{L}_{\mathrm{IR}}+\mathcal{L}_{\mathrm{UV}}\right) .
$$

The anisotropic scaling in the UV limit (1.1) lets the time specific and reduces the diffeomorphisms of GR to foliation-preserving diffeomorphisms on the hypersurfaces of constant time. Thus the Arnowitt-Deser-Misner decomposition of the metric is well equipped

$$
d s^{2}=-N^{2} d t^{2}+g_{i j}\left(d x^{i}+N^{i} d t\right)\left(d x^{j}+N^{j} d t\right) .
$$

The IR Lagrangian density is easily determined by requiring GR as the IR fixed point

$$
\mathcal{L}_{\mathrm{IR}}=\alpha\left(K_{i j} K^{i j}-\lambda K^{2}\right)+\xi R+\sigma,
$$


where the extrinsic curvature is given by

$$
K_{i j} \equiv \frac{1}{2 N}\left(\dot{g_{i j}}-\nabla_{i} N_{j}-\nabla_{j} N_{i}\right), \quad K=g^{i j} K_{i j}, \quad K^{i j}=g^{i k} g^{j l} K_{k l}, \quad \dot{g_{i j}} \equiv \frac{\partial g_{i j}}{\partial t} .
$$

In this GR limit with general covariance, the 4 parameters in (2.3) have the fixed values by the Newton's constant $G$, the speed of light $c=1$, and the cosmological constant $\Lambda$ as

$$
\lambda=1, \quad \alpha=\frac{1}{16 \pi G c}, \quad \xi=\frac{c}{16 \pi G}, \quad \sigma=-\frac{c \Lambda}{8 \pi G} .
$$

Since the $z=3$ scaling (1.1) still allows 5 sixth order spatial derivative terms [6], inclusion of all the terms makes the theory less predictable. Suppose the detailed balance condition emerges near the UV fixed point and the tensor structure of sixth order spatial derivative terms in the action is determined by the Cotton tensor [1]

$$
C_{i j}=\frac{\epsilon^{i k l}}{\sqrt{g}} \nabla_{k}\left(R^{j}{ }_{l}-\frac{1}{4} R \delta_{l}^{j}\right) .
$$

The relevant deformations need not follow the detailed balance and then the UV Lagrangian density has 4 higher spatial derivative terms

$$
\mathcal{L}_{\mathrm{UV}}=\beta C_{i j} C^{i j}+\gamma \frac{\epsilon^{i j k}}{\sqrt{g}} R_{i l} \nabla_{j} R_{k}^{l}+\zeta R_{i j} R^{i j}+\eta R^{2} .
$$

Though the physical motivation for the introduction of the detailed balance condition is still challenging [5], 16], the assignment of it restricts the number of parameters in the Lagrangian densities in (2.3) and (2.7) to 8 parameters given in descending order of the number of derivatives

$$
\beta \rightarrow \gamma \rightarrow(\zeta, \eta) \rightarrow(\lambda, \alpha, \xi) \rightarrow \sigma
$$

Reflecting the difficulty in the quantization procedure [7], we adopt the projectability condition in which the lapse function $N$ depends only on the time coordinate $t$. Then the time reparametrization, a symmetry transformation in the projectable version of HL gravity, fixes the lapse function to be unity, $N=1$. Since we are interested in stringy static objects, we assume rotational symmetry in the $(r, \theta)$ plane and take the Painlevé-Gullstrand type coordinates of which the metric is compatible with the projectability condition

$$
d s^{2}=-d t^{2}+e^{-2 f(r)}[d r+n(r) d t]^{2}+r^{2} d \theta^{2}+e^{2 h(r)} d z^{2}
$$


Inserting this metric into the HL action (2.1), we have

$$
\begin{aligned}
S_{\mathrm{HL}}= & 2 \pi \int d t d r d z r e^{h-f}\left\{\alpha(1-\lambda)\left(n^{\prime 2}-2 n n^{\prime} f^{\prime}+n^{2} f^{\prime 2}+n^{2} h^{\prime 2}+\frac{n^{2}}{r^{2}}\right)\right. \\
& -2 \alpha \lambda\left[\left(n n^{\prime}-n^{2} f^{\prime}\right)\left(\frac{1}{r}+h^{\prime}\right)+\frac{n^{2} h^{\prime}}{r}\right] \\
& +\frac{\beta}{2} e^{4 f}\left[\frac{h^{\prime}-f^{\prime}}{r^{2}}+\frac{f^{\prime \prime}-h^{\prime \prime}+2\left(f^{\prime 2}-h^{\prime 2}\right)}{r}-2 f^{\prime 2} h^{\prime}-3 f^{\prime} h^{\prime \prime}-2 f^{\prime} h^{\prime 2}-f^{\prime \prime} h^{\prime}-2 h^{\prime} h^{\prime \prime}-h^{\prime \prime \prime}\right]^{2} \\
& +\zeta e^{4 f}\left[\left(\frac{f^{\prime}}{r}+f^{\prime} h^{\prime}+h^{\prime \prime}+h^{\prime 2}\right)^{2}+\left(\frac{f^{\prime}}{r}+\frac{h^{\prime}}{r}\right)^{2}+\left(\frac{h^{\prime}}{r}+f^{\prime} h^{\prime}+h^{\prime \prime}+h^{\prime 2}\right)^{2}\right] \\
& \left.+4 \eta e^{4 f}\left(\frac{f^{\prime}}{r}+\frac{h^{\prime}}{r}+f^{\prime} h^{\prime}+h^{\prime \prime}+h^{\prime 2}\right)^{2}-2 \xi e^{2 f}\left(\frac{f^{\prime}}{r}+\frac{h^{\prime}}{r}+f^{\prime} h^{\prime}+h^{\prime \prime}+h^{\prime 2}\right)+\sigma\right\}, \quad(2.10)
\end{aligned}
$$

where the prime' denotes $d / d r$. Note that the parity-violating fifth order derivative term in (2.7) vanishes, and then the action (2.10) involves 7 parameters except $\gamma$. For later convenience we introduce the action of matter fields,

$$
S_{\mathrm{M}}=2 \pi \int d t d r d z r e^{h-f} \mathcal{L}_{\mathrm{M}}(n, f, h)
$$

The equations of motion are read by varying the actions (2.10)-(2.11) with respect to the metric functions. The variation of $n$ gives

$$
\begin{aligned}
& (1-\lambda)\left[1+r^{2} f^{\prime \prime}-\frac{r n^{\prime}}{n}\left(1-r f^{\prime}+r h^{\prime}\right)-\frac{r^{2} n^{\prime \prime}}{n}\right]+\lambda r^{2} h^{\prime \prime}+r f^{\prime}\left(1+r h^{\prime}\right)+r^{2} h^{\prime 2} \\
& =-\frac{r^{2}}{2 \alpha n} \frac{\partial \mathcal{L}_{\mathrm{M}}}{\partial n}
\end{aligned}
$$

but we omit the equations of $f$ and $h$, which are too lengthy.

In order to obtain exact solutions under minimal restriction, we fix a parameter among the remaining 7 parameters. Specifically, reflecting the revival of general covariance in the IR limit, we choose

$$
\lambda=1
$$

In HL gravity possessing an extra U(1) gauge symmetry [2], the fixation of (2.13) is naturally forced. If some exact solutions are obtained under this choice, those are valid in the IR regime. Under (2.13), the Eq. (2.12) is simplified

$$
f^{\prime}=-\frac{r}{1+r h^{\prime}}\left(h^{\prime \prime}+h^{\prime 2}+\frac{1}{2 \alpha n} \frac{\partial \mathcal{L}_{\mathrm{M}}}{\partial n}\right),
$$


however the other equations of $f$ and $h$ are still left in complicated forms

$$
\begin{aligned}
& -2 r^{5} \sigma-4 r^{4} \alpha n n^{\prime}\left(1+r h^{\prime}\right)-4 r^{4} \alpha n^{2}\left(h^{\prime}+r h^{2}+r h^{\prime \prime}\right)-4 e^{4 f} r^{4} \beta h^{\prime 5} \\
& +e^{4 f}\left\{2 r^{2} \beta f^{\prime 3}\left(1-r h^{\prime}\right)\left[r^{2}\left(2 h^{\prime 2}+h^{\prime \prime}\right)-1\right]-2 r^{3} h^{\prime 4}\left[r^{2} \beta\left(f^{\prime \prime}+2 h^{\prime \prime}\right)-5 \beta-2 r^{2}(\zeta+2 \eta)\right]\right. \\
& +2 r^{2} h^{\prime 3}\left[-3 \beta-2 r^{2} \zeta+r^{2} \beta\left(4 f^{\prime \prime}+h^{\prime \prime}+2 r f^{\prime \prime \prime}-r h^{\prime \prime \prime}\right)\right]-r f^{\prime 2}\left[-5 \beta+12 r^{2}(\zeta+2 \eta)\right. \\
& +r\left[8 r^{3} \beta h^{\prime 4}-12 r^{2} \beta h^{\prime 3}+2 r h^{\prime 2}\left(13 \beta+6 r^{2}(\zeta+2 \eta)+r^{2} \beta\left(-9 f^{\prime \prime}+2 h^{\prime \prime}\right)\right)+r \beta\left[-18 f^{\prime \prime}\right.\right. \\
& \left.\left.\left.+h^{\prime \prime}\left(20+19 r^{2} h^{\prime \prime}\right)+14 r h^{\prime \prime \prime}\right]+2 h^{\prime}\left(\beta+6 r^{2}(\zeta+4 \eta)+r^{2} \beta\left(18 f^{\prime \prime}+7 h^{\prime \prime}-7 r h^{\prime \prime \prime}\right)\right)\right]\right] \\
& +r h^{\prime 2}\left[-7 \beta+8 r^{2}(\zeta+\eta)+r^{2}\left[9 r^{2} \beta f^{\prime \prime 2}+4 f^{\prime \prime}\left(-2\left(2 \beta+r^{2}(\zeta+2 \eta)\right)+3 r^{2} \beta h^{\prime \prime}\right)\right.\right. \\
& \left.\left.-2\left(\left(9 \beta+4 r^{2}(\zeta+2 \eta)\right) h^{\prime \prime}+2 r^{2} \beta h^{\prime \prime 2}+r \beta\left(2 f^{\prime \prime \prime}-5 h^{\prime \prime \prime}-r\left(f^{\prime \prime \prime \prime}+2 h^{\prime \prime \prime \prime}\right)\right)\right)\right]\right] \\
& -2 f^{\prime}\left[3 \beta-4 r^{2}(\zeta+2 \eta)\left(1-r^{3} h^{\prime 3}\right)+r\left[2 r^{4} \beta h^{\prime 5}-2 r^{3} \beta h^{\prime 4}+r^{2} h^{\prime 3}\left(9 \beta-r^{2} \beta\left(4 f^{\prime \prime}-7 h^{\prime \prime}\right)\right)\right.\right. \\
& +r h^{2}\left(-9 \beta+8 r^{2}(\zeta+3 \eta)-r^{2} \beta\left(-4 f^{\prime \prime}+7 h^{\prime \prime}+6 r\left(f^{\prime \prime \prime}+h^{\prime \prime \prime}\right)\right)\right)+r\left(12 r^{2} \beta h^{\prime \prime 2}\right. \\
& \left.+\beta f^{\prime \prime}\left(7+11 r^{2} h^{\prime \prime}\right)+h^{\prime \prime}\left(4 r^{2}(\zeta+4 \eta)-7 \beta+7 r^{3} \beta h^{\prime \prime \prime}\right)+r \beta\left(7 h^{\prime \prime \prime}-6 f^{\prime \prime \prime}+5 r h^{\prime \prime \prime \prime}\right)\right) \\
& +h^{\prime}\left(-\beta+4 r^{2}(\zeta+2 \eta)+r^{2}\left(25 \beta+8 r^{2}(\zeta+2 \eta)\right) h^{\prime \prime}+4 r^{4} \beta h^{\prime \prime 2}-r^{2} \beta f^{\prime \prime}\left(7+11 r^{2} h^{\prime \prime}\right)\right. \\
& \left.\left.\left.+r^{3} \beta\left(12 f^{\prime \prime \prime}+6 h^{\prime \prime \prime}-5 r h^{\prime \prime \prime \prime}\right)\right)\right]\right]-r^{3}\left(9 \beta-4 r^{2}(\zeta+2 \eta)\right) h^{\prime \prime 2}-9 r^{3} \beta f^{\prime \prime 2}+4 r^{5} \beta h^{\prime \prime 3} \\
& +2 r f^{\prime \prime}\left(-3 \beta+4 r^{2}(\zeta+2 \eta)+r^{2} \beta\left(6 h^{\prime \prime}+4 r^{2} h^{\prime \prime 2}+7 r h^{\prime \prime \prime}\right)\right) \\
& +2 r h^{\prime \prime}\left(3 \beta+2 r^{2}(\zeta+4 \eta)+r^{3} \beta\left(4 f^{\prime \prime \prime}+8 h^{\prime \prime \prime}+r h^{\prime \prime \prime \prime}\right)\right) \\
& \left.+r^{2}\left[\left(4 r^{2}(\zeta+4 \eta)-6 \beta\right) h^{\prime \prime \prime}+4 \beta f^{\prime \prime \prime}+r^{3} \beta h^{\prime \prime \prime 2}-2 r \beta\left(f^{\prime \prime \prime \prime}-2 h^{\prime \prime \prime \prime}-r h^{\prime \prime \prime \prime \prime}\right)\right]\right\} \\
& +2 e^{2 f} h^{\prime}\left\{2 r^{4} \xi+e^{2 f}\left[3 \beta+2 r^{2}(\zeta+4 \eta)+r^{2}\left[-9 r^{2} \beta f^{\prime \prime 2}-2 r^{2} \beta h^{\prime \prime 2}+r^{3} \beta\left(2 f^{\prime \prime \prime}+h^{\prime \prime \prime}\right)\right.\right.\right. \\
& +2 h^{\prime \prime}\left(4\left(\beta-r^{2}(\zeta+3 \eta)\right)\right)+f^{\prime \prime}\left(-2\left(\beta+2 r^{2}(\zeta+4 \eta)\right)+r^{2} \beta\left(-8 h^{\prime \prime}+7 r h^{\prime \prime \prime}\right)\right) \\
& \left.\left.\left.+r\left(2 \beta f^{\prime \prime \prime}-\left(11 \beta+4 r^{2}(\zeta+2 \eta)\right) h^{\prime \prime \prime}-r \beta\left(2 f^{\prime \prime \prime \prime}+h^{\prime \prime \prime \prime}-r h^{\prime \prime \prime \prime \prime}\right)\right)\right]\right]\right\} \\
& =2 r^{5}\left(\mathcal{L}_{\mathrm{M}}-\frac{\partial \mathcal{L}_{\mathrm{M}}}{\partial f}\right)
\end{aligned}
$$




$$
\begin{aligned}
& 2 r^{5}\left(\sigma+2 \alpha n^{\prime 2}\right)+2 r^{4} \alpha n^{2}\left(-2 f^{\prime}+2 r f^{\prime 2}-2 r f^{\prime \prime}\right)+4 r^{4} \alpha n\left(\left(2-3 r f^{\prime}\right) n^{\prime}+r n^{\prime \prime}\right) \\
& -2 e^{4 f} r^{3} h^{\prime 4}\left(5 \beta+2 r^{2}(\zeta+2 \eta)-r^{2} \beta\left(3 f^{\prime \prime}+2 h^{\prime \prime}\right)\right)-4 e^{4 f} r^{3} \beta f^{\prime 4}\left(11+20 r^{2} h^{\prime \prime}+r h^{\prime}\left(10 r h^{\prime}-1\right)\right) \\
& -2 e^{4 f} r^{2} h^{\prime 3}\left(2 r^{2}(\zeta+4 \eta)-8 \beta+r^{2} \beta\left(13 f^{\prime \prime}-7 h^{\prime \prime}+r\left(f^{\prime \prime \prime}-3 h^{\prime \prime \prime}\right)\right)\right)+4 e^{4 f} r^{4} \beta h^{\prime 5} \\
& +24 e^{4 f} r^{4} \beta f^{\prime 5}\left(1-r h^{\prime}\right)+2 e^{4 f} r^{2} f^{\prime 3}\left\{12\left(2 \beta+r^{2}(\zeta+4 \eta)\right)-r\left[44 r \beta h^{\prime 2}+2 r^{2} \beta h^{\prime 3}\right.\right. \\
& \left.\left.+h^{\prime}\left(r^{2} \beta\left(64 f^{\prime \prime}+81 h^{\prime \prime}\right)-3\left(7 \beta+8 r^{2}(\zeta+2 \eta)\right)\right)+r \beta\left(-64 f^{\prime \prime}+22 h^{\prime \prime}+51 r h^{\prime \prime \prime}\right)\right]\right\} \\
& +e^{4 f} r f^{\prime 2}\left\{-41 \beta-8 r^{2}(\zeta+5 \eta)+r\left[-64 r^{2} \beta h^{\prime 3}+16 r^{3} \beta h^{\prime 4}+2 r h^{\prime 2}\left(35 \beta+22 r^{2}(\zeta+2 \eta)\right.\right.\right. \\
& \left.-r^{2} \beta\left(59 f^{\prime \prime}+14 h^{\prime \prime}\right)\right)-2 h^{\prime}\left(28 \beta-r^{2}(26 \zeta+64 \eta)+r^{2} \beta\left(7 f^{\prime \prime}+127 h^{\prime \prime}+37 r f^{\prime \prime \prime}+65 r h^{\prime \prime \prime}\right)\right) \\
& +r\left(\left(68 \beta+88 r^{2}(\zeta+2 \eta)\right) h^{\prime \prime}-22 \beta f^{\prime \prime}\left(5+11 r^{2} h^{\prime \prime}\right)-r \beta\left(70 h^{\prime \prime \prime}+62 r h^{\prime \prime \prime \prime}-74 f^{\prime \prime \prime}\right)\right. \\
& \left.\left.\left.-111 r^{2} \beta h^{\prime \prime 2}\right)\right]\right\}-e^{4 f} r h^{\prime 2}\left\{r ^ { 2 } \left[27 r^{2} \beta f^{\prime \prime 2}+2 f^{\prime \prime}\left(-12 \beta-8 r^{2}(\zeta+2 \eta)+7 r^{2} \beta h^{\prime \prime}\right)\right.\right. \\
& \left.+2\left(\left(17 \beta+4 r^{2}(\zeta+2 \eta)\right) h^{\prime \prime}-6 r^{2} \beta h^{\prime \prime 2}+r \beta\left(11 f^{\prime \prime \prime}+13 h^{\prime \prime \prime}+r\left(3 f^{\prime \prime \prime \prime}+h^{\prime \prime \prime \prime}\right)\right)\right)\right] \\
& \left.+5 \beta+4 r^{2}(\zeta+2 \eta)\right\}-2 e^{4 f} h^{\prime}\left\{r ^ { 2 } \left[h^{\prime \prime}\left(2\left(\beta-r^{2}(5 \zeta+4 \eta)\right)+r^{3} \beta\left(20 f^{\prime \prime \prime}+7 h^{\prime \prime \prime}\right)\right)\right.\right. \\
& +4 r^{2} \beta f^{\prime \prime 2}+21 r^{2} \beta h^{\prime \prime 2}+f^{\prime \prime}\left(2\left(5 \beta-r^{2}(5 \zeta+12 \eta)\right)+r^{2} \beta\left(58 h^{\prime \prime}+17 r f^{\prime \prime \prime}+29 r h^{\prime \prime \prime}\right)\right) \\
& \left.+r^{2} \beta\left(f^{\prime \prime \prime \prime \prime}+13 h^{\prime \prime \prime \prime}+r\left(f^{\prime \prime \prime \prime \prime \prime}+3 h^{\prime \prime \prime \prime \prime}\right)\right)-r\left(3 \beta+4 r^{2}(\zeta+2 \eta)\right)\left(f^{\prime \prime \prime}-2 h^{\prime \prime \prime}\right)\right] \\
& \left.+9 \beta-4 r^{2}(\zeta+2 \eta)\right\}+2 e^{2 f} f^{\prime}\left\{e^{2 f}\left(9 \beta+2 r^{2}(\zeta+4 \eta)\right)-2 r^{4} \xi+e^{2 f} r\left[4 r^{3} \beta h^{\prime 4}+2 r^{4} \beta h^{\prime 5}\right.\right. \\
& -r^{2} h^{\prime 3}\left(13 \beta+4 r^{2}(\zeta+2 \eta)+r^{2} \beta\left(4 f^{\prime \prime}-17 h^{\prime \prime}\right)\right)-r h^{2}\left(8 \beta-4 r^{2}(3 \zeta+2 \eta)\right. \\
& \left.+r^{2} \beta\left(62 f^{\prime \prime}+64 h^{\prime \prime}+22 r f^{\prime \prime \prime}+7 r h^{\prime \prime \prime}\right)\right)-h^{\prime}\left[-23 \beta+12 r^{2}(\zeta+2 \eta)+r^{2}\left[47 r^{2} \beta f^{\prime \prime 2}\right.\right. \\
& -3\left(13 \beta+12 r^{2}(\zeta+2 \eta)\right) h^{\prime \prime}+15 r^{2} \beta h^{\prime \prime 2}+f^{\prime \prime}\left(-23 \beta-28 r^{2}(\zeta+2 \eta)+113 r^{2} \beta h^{\prime \prime}\right) \\
& \left.\left.+r \beta\left(6 f^{\prime \prime \prime}+70 h^{\prime \prime \prime}+10 r f^{\prime \prime \prime \prime}+23 r h^{\prime \prime \prime \prime}\right)\right]\right]+r\left[-57 r^{2} \beta h^{\prime \prime 2}+h^{\prime \prime}\left(-25 \beta+2 r^{2}(15 \zeta+32 \eta)\right.\right. \\
& \left.-5 r^{3} \beta\left(9 f^{\prime \prime \prime}+11 h^{\prime \prime \prime}\right)\right)+f^{\prime \prime}\left(31 \beta+14 r^{2}(\zeta+4 \eta)-r^{2} \beta\left(45 h^{\prime \prime}+73 r h^{\prime \prime \prime}\right)\right)+47 r^{2} \beta f^{\prime \prime 2} \\
& \left.\left.\left.+r\left(-17 \beta f^{\prime \prime \prime}+6\left(3 \beta+4 r^{2}(\zeta+2 \eta)\right) h^{\prime \prime \prime}+r \beta\left(10 f^{\prime \prime \prime \prime}-9\left(2 h^{\prime \prime \prime \prime}+r h^{\prime \prime \prime \prime \prime}\right)\right)\right)\right]\right]\right\}
\end{aligned}
$$




$$
\begin{aligned}
& -e^{4 f} r\left\{2 h^{\prime \prime}\left(4 r^{2}(\zeta+2 \eta)-9 \beta+r^{3} \beta\left(10 f^{\prime \prime \prime}+29 h^{\prime \prime \prime}+6 r f^{\prime \prime \prime \prime}+9 r h^{\prime \prime \prime \prime}\right)\right)\right. \\
& -3 r^{2} h^{\prime \prime 2}\left(3 \beta+4 r^{2}(\zeta+2 \eta)\right)+4 r^{4} \beta h^{\prime \prime 3}+7 r^{2} \beta f^{\prime \prime 2}\left(3+8 r^{2} h^{\prime \prime}\right)+2 f^{\prime \prime}\left[9 \beta+2 r^{2}(\zeta+4 \eta)\right. \\
& \left.+r^{2}\left(-4\left(3 \beta+4 r^{2}(\zeta+2 \eta)\right) h^{\prime \prime}+24 r^{2} \beta h^{\prime \prime 2}+r \beta\left(-17 f^{\prime \prime \prime \prime}+19 h^{\prime \prime \prime}+14 r h^{\prime \prime \prime \prime}\right)\right)\right] \\
& +r\left[4\left(3 \beta-4 r^{2}(\zeta+2 \eta)\right) h^{\prime \prime \prime}+13 r^{3} \beta h^{\prime \prime \prime 2}+2 f^{\prime \prime \prime}\left(-5 \beta-2 r^{2}(\zeta+4 \eta)+13 r^{3} \beta h^{\prime \prime \prime}\right)\right. \\
& \left.\left.+2 r\left(2 \beta f^{\prime \prime \prime \prime}-\left(3 \beta+4 r^{2}(\zeta+2 \eta)\right) h^{\prime \prime \prime \prime}-r \beta\left(f^{\prime \prime \prime \prime \prime}-3 h^{\prime \prime \prime \prime \prime}-r h^{\prime \prime \prime \prime \prime \prime}\right)\right)\right]\right\} \\
& =-2 r^{5}\left(\mathcal{L}_{\mathrm{M}}+\frac{\partial \mathcal{L}_{\mathrm{M}}}{\partial h}\right) .
\end{aligned}
$$

The above equations are derived by variation of the action (2.10), but are checked to be the same as the equations of motion obtained from (2.1) after inserting (2.2) under the projectability condition since the metric (2.9) possesses good symmetries.

\subsection{Exact solution}

From here on, we focus on the case of $\lambda=1$ and obtain some stringy configurations as exact solutions in the absence of matter field, $\mathcal{L}_{\mathrm{M}}=0$. Suppose we have a constant solution of $f$ as

$$
f=\left(\ln f_{0}\right) / 2, \quad\left(f_{0}>0\right) .
$$

Then (2.14) becomes an equation of $h(r)$ and we obtain

$$
h(r)=\ln \left[h_{0}\left(r-r_{0}\right)\right],
$$

where $h_{0}$ and $r_{0}$ are two integration constants, and $h_{0}$ can be set to be unity by a rescaling of the $z$ coordinate. Plugging (2.18) and the constant $f$ solution (2.17) into the remaining two equations (2.15) $-(2.16)$, we find the exact solution for the vanishing integration constant, $r_{0}=0$,

$$
\begin{aligned}
& h(r)=\ln r \\
& n(r)= \pm \sqrt{-\frac{\sigma}{6 \alpha} r^{2}+f_{0} \frac{\xi}{\alpha}+\frac{n_{0}}{r}-\frac{f_{0}^{2}(5 \zeta+14 \eta)}{\alpha r^{2}}}
\end{aligned}
$$

where $n_{0}$ is another integration constant. Note that the Cotton tensor (2.6) vanishes for the obtained solution and it is the reason why the exact solution in (2.17), (2.19), and (2.20) does not involve $\beta$ dependence. Finally we obtain the following metric from (2.9)

$$
d s^{2}=-d t^{2}+\frac{1}{f_{0}}\left(d r \pm \sqrt{-\frac{\sigma}{6 \alpha} r^{2}+f_{0} \frac{\xi}{\alpha}+\frac{n_{0}}{r}-\frac{f_{0}^{2}(5 \zeta+14 \eta)}{\alpha r^{2}}} d t\right)^{2}+r^{2}\left(d \theta^{2}+d z^{2}\right)
$$


where the metric involves a constant metric, $f_{0}$, and an integration constant, $n_{0}$. We will make a few comments on the solution (2.21) and then figure out the physical meaning of those in what follows.

If we regard the $z$ coordinate as a compact one, e.g., an $S^{1}$, the $(\theta, z)$ surface forms a $T^{2}$. In the nonprojectable version of HL gravity involving the lapse function with spatial coordinate dependence, there is another equation for $N(r)$. Then (2.21) need not always be a solution but, actually, is a solution only for the restricted parameters, $3 \zeta+8 \eta=0$. Since the nonprojectable version is a different theory from the projectable version, we shall focus on the solution (2.21) of the projectable HL gravity in this paper.

To compare, under a static metric in the Poincaré coordinates $d s^{2}=-B(r) e^{2 \delta(r)} d \tilde{t}^{2}+\left[d r^{2} / B(r)\right]+$ $r^{2}\left(d \theta^{2}+d z^{2}\right)$, we directly solve the Einstein equations without the matter fields and obtain

$$
d s^{2}=-\left(-\frac{\Lambda}{3} r^{2}-\frac{G M}{r}\right) d \tilde{t}^{2}+\frac{d r^{2}}{-\frac{\Lambda}{3} r^{2}-\frac{G M}{r}}+r^{2}\left(d \theta^{2}+d z^{2}\right),
$$

where $\Lambda$ is a cosmological constant and $M$ is an integration constant. Understanding the physical meaning of the 5 parameters in the solution (2.21) $, \sigma, \xi, \alpha, f_{0}$, and $n_{0}$, we perform the coordinate transformation,

$$
d \tilde{t}=\frac{1}{\sqrt{F_{0}}}\left(d t \mp \frac{\sqrt{F_{0}-B}}{B} d r\right), \quad\left(F_{0}>0\right),
$$

and the resultant metric is

$$
d s^{2}=-d t^{2}+\frac{1}{F_{0}}\left(d r \pm \sqrt{\frac{\Lambda}{3} r^{2}+F_{0}+\frac{G M}{r}} d t\right)^{2}+r^{2}\left(d \theta^{2}+d z^{2}\right) .
$$

Let us take the IR limit of vanishing higher derivative terms in (2.21), $\zeta=\eta=0$, and compare it with (2.24).

Comparing the first terms proportional to $r^{2}$ in (2.21) and (2.24), the identification in the GR limit, $\sigma / 6 \alpha=-\Lambda / 3$, matches the relations in (2.5) and then the first term is interpreted as a cosmological constant term. There is an $r^{2}$ factor in front of $d z^{2}$, and this warp factor seems unavoidable for straight stringy solutions in $(1+3)$-dimensional HL gravity [17]. In addition it makes the integration constant term inversely proportional to the radial coordinate, $-n_{0} / r$, and this $1 / r$ behavior is formally nothing but the mass term in the $(1+3)$-dimensional Schwarzschild solution with spherical symmetry. The metric function in (2.22) does not have a constant term and thus, in anti-de Sitter (AdS) spacetime, it naturally possesses a horizon at $r_{\mathrm{H}}=(-3 G M / \Lambda)^{1 / 3} \sim$ $\left(6 \alpha n_{0} / \sigma\right)^{1 / 3}$ for positive mass $M>0$ similar to the case of the BTZ black hole [18. Therefore, in the GR limit, the integration constant of the $1 / r$ term in (2.24) is identified with the usual mass parameter in (2.22) as $n_{0}=G M$. It can also be confirmed by computing the Kretschmann invariant for (2.22), which shows a physical singularity at the origin for nonvanishing $G M$

$$
R^{\mu \nu \rho \sigma} R_{\mu \nu \rho \sigma}=\frac{12 G^{2} M^{2}}{r^{6}}+\frac{8 \Lambda^{2}}{3} .
$$


Though there looks to be a constant piece $F_{0}$ in (2.24), it is an artifact of the coordinate transformation (2.23) in GR and thus it does not appear as the original metric in (2.22). In HL gravity, on the other hand, the transformation which mixes the $t$ and $r$ coordinates is not allowed as a symmetry transformation. Thus, $f_{0}$ in the HL metric (2.21) should be treated as another integration constant.

From here on we consider an IR regime where the 2 parameters, $\alpha, \xi$, are slightly away from GR but higher spatial derivative terms are left to vanish, $\zeta=\eta=0$. Since we only have an exact solution for $\lambda=1$, we keep $\lambda$ to be unity for tractability. In the metric (2.24) a small variation of the coefficients in the $r^{2}$ and $1 / r$ terms induces only slightly and qualitatively the same change in (2.22). On the other hand, a tiny deviation of $\xi / \alpha$ from unity changes the situation drastically and qualitatively provided the integration constant is not fine-tuned to an extremely small value, $f_{0} \nrightarrow 0^{+}$.

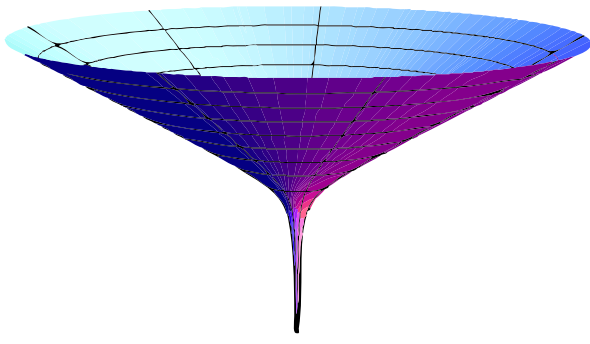

(a)

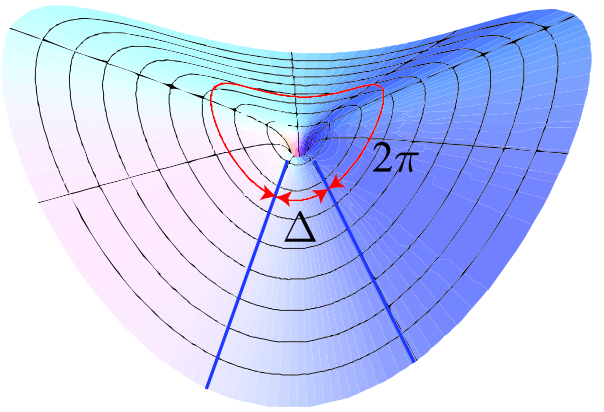

(b)

Figure 1: Surfaces of the $(r, \theta)$ coordinates for vanishing cosmological constant $\sigma=0$, involving a singularity at the origin $r=0$ for $n_{0}>0$. (a) An asymptotic cone of deficit angle $\Delta=2 \pi\left(1-\sqrt{f_{0} \delta}\right)=\pi / 4$ for $0<f_{0} \delta<1$ and $n_{0}=3$. (b) An asymptotic excess cone of surplus angle $\Delta=2 \pi\left(\sqrt{f_{0} \delta}-1\right)=\pi / 4$ for $f_{0} \delta>1$ and $n_{0}=3$.

To illustrate the geometry of this case, let us introduce a parameter $\delta=1-\xi / \alpha$ measuring variation of the graviton speed from unity. First, we consider the parameter region of positive $\delta$ $(\xi / \alpha<1)$, where the graviton speed becomes smaller than unity even in a flat spacetime limit. To make the discussion simpler without spoiling physics we can take the limit of zero cosmological constant $\sigma=0$ and choose zero integration constant $n_{0}=0$ (zero "mass"). Performing the coordinate transformation which preserves the foliation-preserving diffeomorphism and is consistent with the projectability condition,

$$
d t=\sqrt{f_{0}} d \check{t} \pm \frac{\sqrt{\frac{\xi}{\alpha}} d r}{\sqrt{f_{0}}\left(1-\frac{\xi}{\alpha}\right)},
$$


we obtain the metric which is rescaled to be

$$
\begin{aligned}
d s^{2} & =-f_{0} \delta d \check{t}^{2}+\frac{d r^{2}}{f_{0} \delta}+r^{2}\left(d \theta^{2}+d z^{2}\right) \\
& =-d \bar{t}^{2}+d \bar{r}^{2}+\bar{r}^{2}\left(d \bar{\theta}^{2}+d \bar{z}^{2}\right),
\end{aligned}
$$

where the coordinates in the first and second lines are related by

$$
d \check{t}=\frac{d \bar{t}}{\sqrt{f_{0} \delta}}, \quad d r=\sqrt{f_{0} \delta} d \bar{r}, \quad d \theta=\frac{d \bar{\theta}}{\sqrt{f_{0} \delta}} \quad\left(0 \leq \bar{\theta}<2 \pi \sqrt{f_{0} \delta}\right), \quad d z=\frac{d \bar{z}}{\sqrt{f_{0} \delta}} .
$$

For $f_{0}$ satisfying $0<f_{0} \delta<1$, the $(\bar{r}, \bar{\theta})$-space is conic with deficit angle $\Delta=2 \pi\left(1-\sqrt{f_{0} \delta}\right)$. For $f_{0} \delta$ greater than unity, it becomes an excess cone with surplus angle $\Delta=2 \pi\left(\sqrt{f_{0} \delta}-1\right)$. This can also be confirmed by analyzing the geodesic equation of a test body from the metric (2.21). The orbit equation for $u \equiv 1 / r$ corresponding to (2.21) in the limit of $\zeta=\eta=\sigma=n_{0}=0$ is

$$
\frac{d^{2} u}{d \theta^{2}}+\left(f_{0} \delta\right) u=0
$$

and the orbit,

$$
u(\theta)=u_{0} \cos \left(\sqrt{f_{0} \delta} \theta\right)
$$

is closed not at $2 \pi$ but at $2 \pi \sqrt{f_{0} \delta}$. It exactly coincides with the deficit (surplus) angle read from the metric (2.28).

If we turn on nonvanishing positive $n_{0}$, there is the aforementioned singularity at the origin but the $(r, \theta)$ plane approaches a cone (an excess cone) with deficit (surplus) angle in its asymptote irrespective of the value of $n_{0}$. See Fig. 1 from (2.21) with $\sigma=0, f_{0} \delta>0$, and $n_{0}>0$. In the limit of $f_{0} \delta \rightarrow 1$ and $n_{0} \rightarrow 0$, both the deficit and surplus angles approach zero and the geometry of $(r, \theta)$ coordinates is nothing but a flat plane. If the $z$ coordinate is compact like an $S^{1}$, both the $(r, \theta)$ and $(r, z)$ planes form cones with a deficit (surplus) angle.

Astrophysical implication of deficit or surplus angle from a straight stringy object is simple and can easily be detected if the size of the angle is much larger than the known astronomical bound which is about $200 \mu \operatorname{arcs}\left(\sim 10^{-9} \mathrm{rad}\right)$ [19]. When a conic geometry with deficit angle is formed, the light from a star behind the singular stringy source at the apex propagates straight and arrives at a static observer who detects double images projected behind the source [20]. If a cosmic string traverses a star behind it, the light curve increases up by a factor of 2 due to microlensing by the string during the period of traversing [21]. In the case of an excess conic geometry with surplus angle, under the same situation as the deficit angle, the static observer tracking down the trajectory of the star experiences a sudden disappearance of its image for a while and reappearance at a distant point over the source of the surplus angle [12]. The geometry we are dealing with involves an undetermined constant solution $f_{0}$ (2.17), for which there is no way to determine its value or range at the moment. We can only say that its effect may be detected 
for some $f_{0}$ sufficiently larger than $1 / \delta$, which must be very large near GR. Though there does not seem to be any report on the astronomical observation of the surplus angle in the physics world governed by GR and its Newtonian limit, we are actually surrounded by surplus angles on the leaves in our daily life or in the world of plants [see Fig. 1 (b) of which the $n_{0} \rightarrow 0$ limit can be viewed as a leaf of locus].

Second, we consider the parameter region of negative $\delta(\xi / \alpha>1)$, where the graviton speed becomes larger than unity even in the flat spacetime limit. To keep the correct spacetime signature at asymptotic space, we choose anti-de Sitter spacetime of a negative cosmological constant $\sigma>$ $0(\Lambda<0)$ but again turn off the mass $n_{0}=0(G M=0)$ for simplicity. Then, in a $(1+2)$ dimensional spacetime of $d z=0$, the metric,

$$
d s^{2}=-d t^{2}+\frac{1}{f_{0}}\left(d r \pm \sqrt{-\frac{\sigma}{6 \alpha} r^{2}+f_{0} \frac{\xi}{\alpha}} d t\right)^{2}+r^{2} d \theta^{2},
$$

describes a BTZ black hole with a horizon at $r_{\mathrm{H}}=\sqrt{6 \alpha f_{0}|\delta| / \sigma}$, which moves to zero in the limit of GR. In $(1+3)$ dimensions, (2.32) with $r^{2} d z^{2}$ describes a straight black string.

In the context of GR the planar geometry of a cone (an excess cone) is generated by a $\delta$-function source of positive (negative) energy density. In three spatial dimensions the conic geometry is obtained by assuming a thin infinitely stretched source, and it is applied for depicting cosmic strings in the early universe [20]. In HL gravity we find a solution of constant $f$ (2.17) and logarithmic $h$ (2.19) under a physically allowable parameter (2.13). Then, for $\zeta=\eta=0$ and $\sigma=0$, the only equation for the shift function $n(r) / f_{0}$ reduces to

$$
\left(r n^{2}\right)^{\prime}=f_{0} \frac{\xi}{\alpha},
$$

and it supports the conic (excess conic) geometry with deficit (surplus) angle in (2.28) as a static vacuum solution. Let us try to obtain the same solution in the context of GR. We assume the metric with undetermined shift function,

$$
d s^{2}=-d t^{2}+\frac{1}{f_{0}}[d r+n(r) d t]^{2}+r^{2}\left(d \theta^{2}+d z^{2}\right),
$$

and then the Einstein equation, $G^{\mu}{ }_{\nu}=8 \pi G T^{\mu}{ }_{\nu}$, becomes

$$
\left(r n^{2}\right)^{\prime}=f_{0}-8 \pi G r^{2} T_{t}^{t}
$$

In order to obtain a cone (an excess cone) in the $(r, \theta)$ plane orthogonal to the string with a warp factor along the string direction, $r^{2} d z^{2}$ in GR, we should add the negative energy density

$$
8 \pi G\left(-T_{t}^{t}\right)=-f_{0} \delta / r^{2}<0 .
$$

Amazingly only the negative energy density of $f_{0} \delta>0$ can generate either the solution of the deficit angle $\left(0<f_{0} \delta<1\right)$ or that of the surplus angle $\left(f_{0} \delta>1\right)$ in GR, which violates the 
positive energy theorem. In HL gravity, on the other hand, we assumed the absence of matter, $\mathcal{L}_{\mathrm{M}}=0$, and thus the obtained solution is generic.

To read the singularity in HL gravity, we look into the curvature tensor $R_{i j k l}$ and the extrinsic curvature $K_{i j}$. Therefore possible candidates can be two scalar quantities invariant under foliationpreserving diffeomorphism, which are a three-dimensional analog of the Kretschmann invariant, $R^{i j k l} R_{i j k l}=4 R_{i j} R^{i j}-R^{2}$, and a square of the extrinsic curvature, $K_{i j} K^{i j}$, which is a kinetic part of the $(1+3)$-dimensional scalar curvature. Substitution of the metric (2.9) and the vacuum solution (2.21) leads to

$$
\begin{aligned}
R^{i j k l} R_{i j k l} & =4 e^{4 f}\left[\frac{f^{\prime 2}+h^{\prime 2}}{r^{2}}+\left(f^{\prime} h^{\prime}+h^{\prime 2}+h^{\prime \prime}\right)^{2}\right] \\
& =\frac{4 f_{0}^{2}}{r^{4}}, \\
K_{i j} K^{i j} & =n^{2}\left[\frac{1}{r^{2}}+\left(f^{\prime}-\frac{n^{\prime}}{n}\right)^{2}+h^{\prime 2}\right] \\
=\frac{1}{r^{2}} & \left\{-\frac{\sigma}{3 \alpha} r^{2}+\frac{2 f_{0} \xi}{\alpha}+\frac{2 n_{0}}{r}-\frac{2 f_{0}^{2}(5 \zeta+14 \eta)}{\alpha r^{2}}+\frac{\left[-\frac{\sigma}{3 \alpha} r^{2}-\frac{n_{0}}{r}+\frac{2 f_{0}^{2}(5 \zeta+14 \eta)}{\alpha r^{2}}\right]^{2}}{-\frac{2 \sigma}{3 \alpha} r^{2}+\frac{4 f_{0} \xi}{\alpha}+\frac{4 n_{0}}{r}-\frac{4 f_{0}^{2}(5 \zeta+14 \eta)}{\alpha r^{2}}}\right\} .
\end{aligned}
$$

Since (2.37) is independent of the shift function $n(r) / f_{0}$, the singularity at $r=0$ originated from the warp factor in front of $d z^{2}$. When $\sigma=\zeta=\eta=0$, the leading singular behavior at short distance is $K_{i j} K^{i j} \sim n_{0} / r^{3}$ which matches $1 / r^{6}$ behavior in (2.25). The subleading singular behavior is $\mathcal{O}\left(1 / r^{2}\right)$ with proportionality constant $f_{0} \xi / \alpha$, and it looks consistent with the matter (2.36) in GR. Note that there exists another singularity at $r=-n_{0} \alpha / f_{0} \xi$ from the last term of (2.40), only when a negative $n_{0}$ is turned on in (2.40) [22].

\subsection{Pattern of RG flows}

From now on let us examine the obtained exact solutions (2.21) near GR with $\zeta=\eta=0$ and explore possible implication to RG flow in the vicinity of the IR fixed point, which satisfies the requirements 1-3 in Sec. 1. Among the 4 parameters in IR regime (2.8) the ratio between two kinetic terms in (2.3),$\lambda$, is assumed to be fixed as unity since the solutions of consideration (2.21) are obtained under $\lambda=1$. Previously $\sigma$ was identified with a cosmological constant and is left to be undetermined except the extremely tiny value of the present Universe. For $\xi$ and $\alpha$, it is convenient to introduce the variable Newton's constant $G_{\mathrm{HL}}$ and the variable speed of graviton $c_{\mathrm{HL}}$ in HL gravity, visualizing easily the variation from their GR values, $G$ and $c=1$, as

$$
\frac{1}{\alpha \xi}=\left(16 \pi G_{\mathrm{HL}}\right)^{2}, \quad \frac{\xi}{\alpha}=c_{\mathrm{HL}}^{2} .
$$

The parameter $\alpha$ in front of the kinetic terms is kept to be positive in order not to make the gravitons the ghosts and then (2.41) forces $\xi$ to be non-negative. When $c_{\mathrm{HL}}^{2}<1$, we have three 


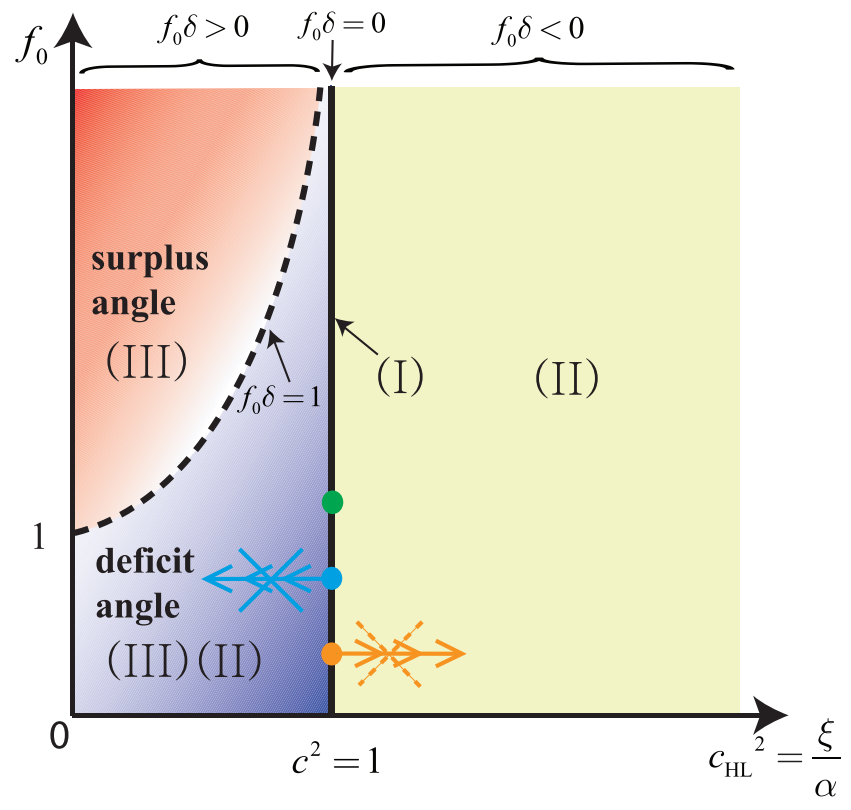

Figure 2: Parameter space of $\left(\xi / \alpha, f_{0}\right)$. The red region (upper left) of $f_{0} \delta>1$ supports excess cones of surplus angle. As the red color becomes thicker, the surplus angle increases to $2 \pi$. The dashed curve of $f_{0} \delta=1$ supports a geometry without deficit/surplus angle. The blue color region (bottom left) of $0<f_{0} \delta<1$ supports cones with deficit angle. As the blue color becomes thicker, the deficit angle increases to $2 \pi$. The thick vertical line of $\delta=0$ denotes the IR fixed point of GR. The ivory region (right) of $\delta<0$ involves the geometry of AdS black string and/or highly curved inner space bounded by a horizon.

solutions of a cone with deficit angle for $f_{0} \delta<1$ (the region of blue color in Fig. 2), an excess cone with surplus angle for $f_{0} \delta>1$ (the region of red color), and the space with zero deficit/surplus angle for $f_{0} \delta=1$ (the boundary of the dashed line). As discussed previously all three solutions are obtained without matter in HL gravity (2.33) but can be obtained with negative energy density in GR (2.36). From the discussion in Sec. 1, these solutions are subject to category (III). Even though a conic geometry with deficit angle can be obtained in GR with a thin filament type cosmic string of positive energy density, the large deficit angle around $2 \pi$ has never been observed, which corresponds to $f_{0} \delta \rightarrow 0^{+}$. Since the flow should start from the IR fixed point of GR with $\delta=0$ (the vertical line of $c_{\mathrm{HL}}^{2}=c^{2}=1$ in Fig. 2), this cone solution belongs to category (II). Therefore, we may say that the RG flow to the direction of decreasing the graviton speed $c_{\mathrm{HL}}^{2}<1$ (the flow line of sky blue color in Fig. 2) seems unlikely and the region of $c_{\mathrm{HL}}^{2}<1$ may belong to an unlikely zone (the regions of red and blue color in Fig. 2). When $c_{\mathrm{HL}}^{2}>1$, the constant piece of metric as in (2.27) can change signature of the time and radial coordinates. It does not occur either in the large distance region of the AdS space $(\sigma>0)$ or in the short distance region of negative mass 
$\left(n_{0}<0\right)$. Though both the outer space of AdS black string and the highly curved inner space bounded by a horizon due to a negative mass are allowable and even familiar theoretically, they have also been hardly detected in the astrophysical observations. Therefore, the region of $c_{\mathrm{HL}}^{2}>1$ also seems to be a candidate of the disfavored zone (II) (the region of ivory color in Fig. 2) and the RG flow may not easily run to this direction (the flow line of orange color). In synthesis, a possible tentative conclusion in the basis of a few tests favors an RG flow in which the graviton speed $c_{\mathrm{HL}}$ remains to be unity (the dot of green color in Fig. (2) but the Newton's constant $G_{\mathrm{HL}}$ varies like $16 \pi G_{\mathrm{HL}}=c_{\mathrm{HL}} / \xi=1 / c_{\mathrm{HL}} \alpha$. The direction of flow to the regime of weak or strong gravitational coupling cannot be determined by the classical solutions of consideration (2.21).

Two comments are in order: First, once $\xi / \alpha$ flows away from $c^{2}=1$ before developing other parameters $\zeta$ and $\eta$ with keeping $\lambda$ to be unity, the corresponding pure HL gravity can possess a formal general coordinate invariance based on the Lorentz symmetry with the changed graviton speed $c_{\mathrm{HL}}=\sqrt{\xi / \alpha} \neq 1$. This kind of accidental Lorentz symmetry can also arise in condensed matter systems, e.g., the low energy effective theory of monolayer graphene with the Fermi speed $v_{\mathrm{F}} \sim c / 300$ [23. Second, near the presumed IR fixed point of GR, both $\xi / \alpha$ and $\lambda$ parameters in (2.3) can run away from unity in general. In this paper, an analysis has always been made by using the solutions with $\lambda=1$ but assumed $\xi / \alpha$ arbitrary so that its application to the IR behavior of RG flow is restricted. Since the structure of highest derivative term in UV regime is based on the detailed balance with the Cotton tensor, an attractive value of $\lambda$ in the UV fixed point is $\lambda=1 / 3$ by assuming anisotropic Weyl symmetry. It may imply a preferred flow path in which $\lambda$ starts from unity, may decrease monotonic, and approaches slowly $1 / 3$.

\section{Charged Black Holes and Flow for Curvature Square Terms}

In this section let us take into account both nonvanishing matter distributions, $\mathcal{L}_{\mathrm{M}} \neq 0$, and higher order curvature terms, $\zeta \neq 0$ and $\eta \neq 0$. We look into their effect from static axially symmetric solutions and discuss similarity between the effect of higher derivative terms and that of the static electric field.

Even though we choose $\lambda$ to be unity (2.13), Eqs. (2.14)-(2.16) are still complicated for nonzero $\zeta$ and $\eta$, and thus we again restrict our interest to the solution of constant $f$ (2.17). When we examine various static stringy matter configurations compatible with the axially symmetric Painlevé-Gullstrand type metric (2.9), the corresponding Lagrangian densities for many of those

are independent of the metric function $n(r), \frac{\partial \mathcal{L}_{\mathrm{M}}}{\partial n}=0$, and then the last matter term on the right-hand side of (2.14) vanishes. It means that the obtained logarithmic solution of $h$ in (2.19) with vanishing integration constant $r_{0}=0$ can also be a solution with the matter. Insertion of $f$ (2.17) and $h$ (2.19) into the remaining equations (2.15) $-(2.16)$ greatly simplifies and reexpresses 
the equations (2.15)-(2.16) as a first order equation for $n$,

$$
\left(r n^{2}\right)^{\prime}=\frac{f_{0}^{2}(5 \zeta+14 \eta)}{\alpha r^{2}}+f_{0} \frac{\xi}{\alpha}-\frac{\sigma}{2 \alpha} r^{2}-\frac{r^{2}}{2 \alpha}\left(\mathcal{L}_{\mathrm{M}}-\frac{\partial \mathcal{L}_{\mathrm{M}}}{\partial f}\right)
$$

and a first order constraint equation for the matter field,

$$
\left(\mathcal{L}_{\mathrm{M}}-\frac{\partial \mathcal{L}_{\mathrm{M}}}{\partial f}\right)^{\prime}=\frac{2}{r}\left(\frac{\partial \mathcal{L}_{\mathrm{M}}}{\partial f}+\frac{\partial \mathcal{L}_{\mathrm{M}}}{\partial h}\right)
$$

which should be consistent with the second order Euler-Lagrange equation from $\mathcal{L}_{\mathrm{M}}$.

\subsection{Exact solution}

As a simple but representative example we consider the electric field produced by an electrically charged thin filament stretched straightly along the $z$-direction. Its dynamics is described by the Lagrangian density

$$
\mathcal{L}_{\mathrm{M}}=\frac{1}{2} e^{2 f} F_{r 0}^{2}
$$

For the field theories with anisotropic scaling in their UV regime, the potential term develops naturally higher spatial derivative terms but a quadratic time derivative structure of the kinetic term, $E_{i}^{2}=F_{i 0}^{2}$, is unaltered along the flow from IR to UV, which is a good property as a candidate of matter field in the context of HL gravity. The profile of the electric field is obtained by solving Gauss's law with an electric source of charge density per unit length $q_{\mathrm{e}}$,

$$
E_{r}=F_{r 0}=e^{-(f+h)} \frac{q_{\mathrm{e}}}{4 \pi r} .
$$

The obtained configurations of $f(2.17), h$ (2.19), and $F_{r 0}$ (3.4) satisfy the constraint (3.2). In the presence of the electrostatic field we find an exact solution by solving the remaining equation (3.1),

$$
n(r)= \pm \sqrt{-\frac{\sigma}{6 \alpha} r^{2}+f_{0} \frac{\xi}{\alpha}+\frac{n_{0}}{r}-\frac{f_{0}^{2}(5 \zeta+14 \eta)}{\alpha r^{2}}-\frac{q_{\mathrm{e}}^{2}}{64 \alpha \pi^{2} r^{2}}} .
$$

If we directly solve the Einstein equations with the same electric field in the Poincaré coordinates, we have

$$
d s^{2}=-\left(-\frac{\Lambda}{3} r^{2}-\frac{G M}{r}+\frac{G q_{\mathrm{e}}^{2}}{4 \pi r^{2}}\right) d \tilde{t}^{2}+\frac{d r^{2}}{-\frac{\Lambda}{3} r^{2}-\frac{G M}{r}+\frac{G q_{\mathrm{e}}^{2}}{4 \pi r^{2}}}+r^{2}\left(d \theta^{2}+d z^{2}\right) .
$$

To compare we perform a coordinate transformation (2.23) and then obtain

$$
d s^{2}=-d t^{2}+\frac{1}{F_{0}}\left(d r \pm \sqrt{\frac{\Lambda}{3} r^{2}+F_{0}+\frac{G M}{r}-\frac{G q_{\mathrm{e}}^{2}}{4 \pi r^{2}}} d t\right)^{2}+r^{2}\left(d \theta^{2}+d z^{2}\right) .
$$


As discussed in the previous section, $\xi / \alpha$ in (3.5) should be chosen as unity in the GR limit and, with the help of relation (2.5), the $1 / r^{2}$ term coincides exactly with the term from the electric field in (3.6) and (3.7).

Since we already discussed physics of the other terms with $\sigma, \xi / \alpha, f_{0}$, and $n_{0}$ in the previous section, we focus on the electric field term with $q_{\mathrm{e}}^{2}$ and the higher spatial derivative terms with $\zeta$ and $\eta$ in (3.5). At the level of classical metric (3.5) both terms are not distinguishable due to the same $1 / r^{2}$ behavior so that it may be natural to introduce an effective electric charge $q_{\text {eff }}$ as

$$
q_{\mathrm{eff}}^{2}=q_{\mathrm{e}}^{2}+64 \pi^{2} f_{0}^{2}(5 \zeta+14 \eta)
$$

This is manifested in the curvatures: the three-dimensional analog of the Kretschmann invariant is unchanged (2.38) and the square of the extrinsic curvature keeps its form (2.40) except for a replacement, $f_{0}^{2}(5 \zeta+14 \eta) \rightarrow q_{\text {eff }}^{2} / 64 \pi^{2}$, which means the leading behavior of singularity at the origin is governed by the effective charge (3.8).

When $q_{\mathrm{eff}}^{2}>0$ or equivalently $5 \zeta+14 \eta>-q_{\mathrm{e}}^{2} / 64 \pi^{2} f_{0}^{2}$, an AdS RN type charged black string can usually be formed. It may be timely to investigate the horizon in (3.5) in comparison with (3.7). We assume positive $n_{0}$ in relation with the positivity of mass in the GR limit. In AdS space with $\sigma>0$ the possible number of horizons are from zero to two for $1-\xi / \alpha>0$. In the limit of vanishing cosmological constant $\sigma \rightarrow 0^{+}$the horizon is explicitly given as

$$
\left\{\begin{array}{ll}
r_{ \pm}=\frac{n_{0}}{2 f_{0}\left(1-\frac{\xi}{\alpha}\right)}\left[1 \pm \sqrt{1-\frac{f_{0} q_{\mathrm{eff}}^{2}}{16 \alpha \pi^{2} n_{0}^{2}}\left(1-\frac{\xi}{\alpha}\right)}\right], & \text { when } \frac{f_{0} q_{\mathrm{eff}}^{2}}{16 \alpha \pi^{2} n_{0}^{2}}\left(1-\frac{\xi}{\alpha}\right)<1 \\
r_{\mathrm{e}}=\frac{n_{0}}{2 f_{0}\left(1-\frac{\xi}{\alpha}\right)}, & \text { when } \frac{f_{0} q_{\mathrm{eff}}^{2}}{16 \alpha \pi^{2} n_{0}^{2}}\left(1-\frac{\xi}{\alpha}\right)=1 . \\
\text { no horizon, } & \text { when } \frac{f_{0} q_{\mathrm{eff}}^{2}}{16 \alpha \pi^{2} n_{0}^{2}}\left(1-\frac{\xi}{\alpha}\right)>1
\end{array} .\right.
$$

The horizon at $r_{-}$is analogous to the additional horizon in the RN black hole, and $r_{\mathrm{e}}$ is the unique horizon in the extremal limit. When $1-\xi / \alpha \rightarrow 0, r_{+}$in (3.9) moves to infinity and a single horizon is left at

$$
r_{\mathrm{H}}=\frac{q_{\mathrm{eff}}^{2}}{64 \alpha \pi^{2} n_{0}}
$$

The above charged (black) string structure is maintained even in the absence of the electric charge $q_{\mathrm{e}}=0$ as long as $5 \zeta+14 \eta$ is positive.

When $q_{\text {eff }}^{2}<0$, this effective charge does not produce an additional horizon but modifies the value of horizon due to mass term $n_{0}$. When $\sigma \rightarrow 0^{+}$and $1-\xi / \alpha>0$, it has

$$
r_{\mathrm{H}}=\frac{n_{0}}{2 f_{0}\left(1-\frac{\xi}{\alpha}\right)}\left[1+\sqrt{1-\frac{f_{0} q_{\mathrm{eff}}^{2}}{16 \alpha \pi^{2} n_{0}^{2}}\left(1-\frac{\xi}{\alpha}\right)}\right] .
$$


Let us try to obtain the solution in the context of GR under the metric assumption (2.34). For vanishing cosmological constant, the Einstein equations reduce to (2.35). Comparing it with (3.1) in the limit of $\xi / \alpha=1, \sigma=0$, and $\mathcal{L}_{\mathrm{M}}=0$, we have $8 \pi G\left(-T_{t}^{t}\right)=f_{0}^{2}(5 \zeta+14 \eta) / \alpha r^{4}$ which leads to a distribution of negative energy density for $5 \zeta+14 \eta<0$. Obtaining this solution in GR violates the positive energy theorem, but it is a generic vacuum solution in HL gravity. In case that the source is assumed to be an electrostatic field, $E_{r}$, then it requires $-T_{t}^{t}=f_{0} E_{r}^{2} / 2<0$. Substituting the solutions (2.17) and (2.19) into (3.4), the energy density has $-T_{t}^{t}=q_{\mathrm{e}}^{2} / 32 \pi^{2} r^{4}$. Therefore, we read the effect of quartic spatial derivative terms as that of electric charge $q_{\mathrm{e}}^{2}=4 \pi f_{0}^{2}(5 \zeta+14 \eta) / G \alpha$ and then $q_{\mathrm{e}}^{2}$ can be interpreted to be negative for $5 \zeta+14 \eta<0$. It is forbidden to let the square of physical electric charge negative, $q_{\mathrm{e}}^{2}<0$ in Maxwell theory, since it means that the classical Coulomb force is attractive between two electric charges of the same sign. The square of effective charge (3.8), on the other hand, can take a negative value for $5 \zeta+14 \eta<-q_{\mathrm{e}}^{2} / 64 \pi^{2} f_{0}^{2}$. Since the kinetic term given by the square of the electric field [24] is kept to be quadratic, the property of attractive Coulomb force between the same charges holds in the Lifshitz type field theories irrespective of the anisotropic scaling in the matter sector.

Since the $1 / r^{2}$ terms from both the higher spatial derivative terms and electric charges are subleading to the $1 / r$ term in the astronomical scale, it is difficult to detect such a feeble astrophysical signal in the long distance physics. Here we have some qualitatively unusual results in the context of GR so it may be worth studying their astrophysical effect originated from indistinguishability between the higher derivative term effect and the electric charge. Astrophysical realization of the charged black holes and strings is believed to be difficult since electric charges repel each other. In the UV regime of HL gravity with higher spatial derivative terms of positive $5 \zeta+14 \eta$, production of the charged black strings seems not to be hindered by the repulsive nature between electric charges. Suppose such charged black strings have sufficiently been generated in the early universe due to the quartic spatial derivative terms and keep their nature along the flow to the present IR regime. Although the assumptions seem very rough, such objects or their indirect astrophysical signals may be observed occasionally in the present universe. In this sense future black hole observations including their detailed properties may provide some possibility to be used to constrain the possible parameter region of $5 \zeta+14 \eta$ as long as one cannot find a compelling reason to set $f_{0}$ to be sufficiently small.

In this section we assumed the same electric field of accumulated charges, and the obtained results depend on the form of metric and the assigned conditions in HL gravity. In the previous work [12], on the other hand, the detailed balance condition was assumed in the entire energy scale but the projectability condition was not. As a result, the electric field from accumulated charges induced a geometry with deficit or surplus angle for $1 / 3 \leq \lambda<1 / 2$. In the present work the projectability condition is assumed but the detailed balance condition is imposed only at the UV fixed point. Then the same electric field source leads to the $1 / r^{2}$ term in the metric for $\lambda=1$, which is the same as the usual $1 / r^{2}$ term in the RN type charged black hole. Between GR and HL gravity, this kind of difference basically originates from higher spatial derivative terms. It also 
shows that HL gravity theories become different with and without the detailed balance condition, even though they share similar higher spatial derivative terms.

\subsection{Pattern of RG flows}

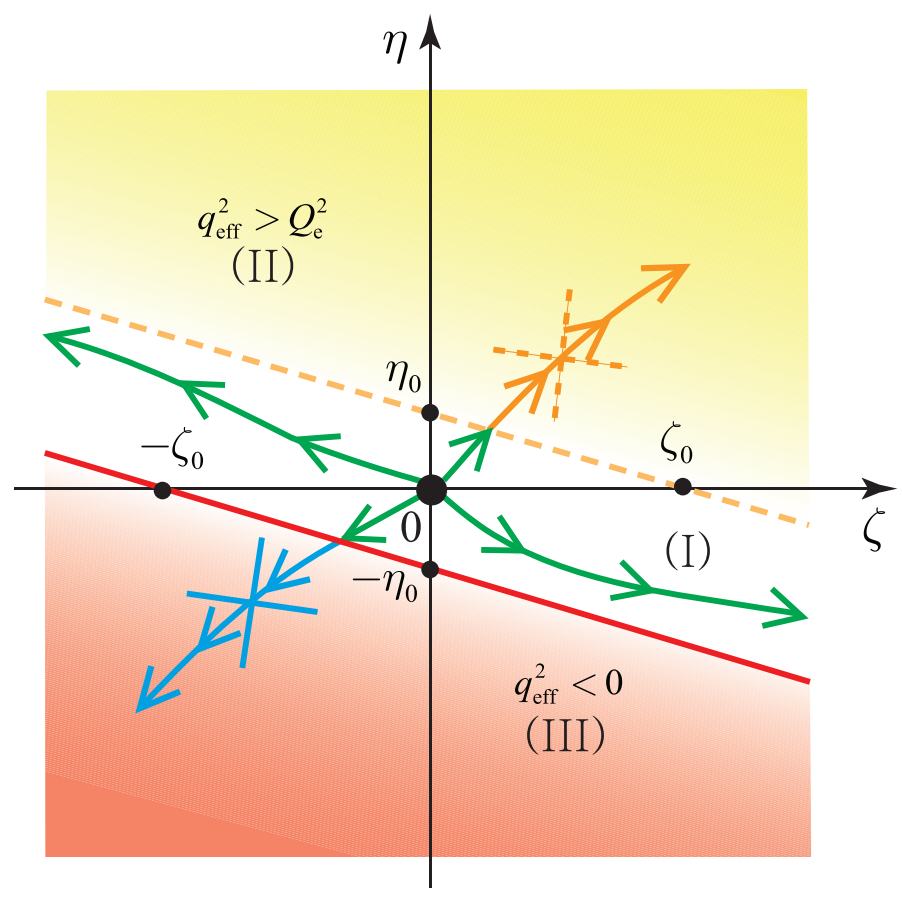

Figure 3: Parameter space of $(\zeta, \eta)$. The nature of Coulomb force between two charges of the same charge is effectively attractive in the red region (lower) of $5 \zeta+14 \eta<-Q_{\mathrm{e}}^{2} / 64 \pi^{2} f_{0}^{2}$. The size of the effective charge is larger than the upper bound $\left|Q_{\mathrm{e}}\right|$ in the yellow region (upper) of $5 \zeta+14 \eta>Q_{\mathrm{e}}^{2} / 64 \pi^{2} f_{0}^{2}$. Here $\zeta_{0}=Q_{\mathrm{e}}^{2} / 896 \pi^{2} f_{0}^{2}$ and $\eta_{0}=Q_{\mathrm{e}}^{2} / 320 \pi^{2} f_{0}^{2}$.

Discussion on the patterns of RG flows utilizing the classical solutions becomes subtle for the coefficients of quartic spatial derivative terms, $\zeta$ and $\eta$ of $R_{i j} R^{i j}$ and $R^{2}$ terms respectively. If nonzero values of $\zeta$ and $\eta$ are developed just above the Planck scale near the IR fixed point, application of the classical solutions may be more reliable. If those coefficients start to develop their nonzero values at some intermediate energy scale which is much larger than the Planck scale but lower than the scale of the Lifshitz fixed point, it is skeptical about the scenario relying heavily on the GR data, theoretical and observational. Coulomb repulsion among the charges of the same sign disfavors the formation of charged astronomical objects. Therefore, we frequently observe neutral objects like almost all the stars and neutron stars. Though the charge of RN type black holes can arbitrarily be large, accumulation of the excess charge by the physical process 
is probably limited by an extremely tiny ratio to mass, and thus, in a statistical sense, we can naturally assume an upper bound of the accumulated charge $Q_{\mathrm{e}}$. As discussed in Sec. 3.1, the square of the effective charge can be negative, $q_{\mathrm{eff}}^{2}<0$, despite positive $q_{\mathrm{e}}^{2}$. Therefore the effect of quartic spatial derivative terms corresponds to the attractive Coulomb force between two charges of the same sign and introduction of negative energy density in GR, which are not allowed in electrodynamics and in GR, respectively. According to the forbidden attractive Coulomb force between two charges of the same sign and violation of the positive energy theorem in GR, the solution belongs to category (III) and the region of negative $q_{\mathrm{eff}}^{2}$ may be an unlikely zone for the RG flows for $\zeta$ and $\eta, 5 \zeta+14 \eta<-Q_{\mathrm{e}}^{2} / 64 \pi^{2} f_{0}^{2}$ (the region of red color and the flow line of sky blue color in Fig. 3). Since an object carrying the electric charge larger than $Q_{\mathrm{e}}$ can rarely be observed, the solution belongs to category (II) and the entrance of RG flows to the region of $5 \zeta+14 \eta>Q_{\mathrm{e}}^{2} / 64 \pi^{2} f_{0}^{2}$ may also be disfavored (the region of yellow color and the flow line of orange color in Fig. 3). Therefore, the RG flows about the line of $5 \zeta+14 \eta=0$ are allowed (the band of white color and the flow lines of green color in Fig. 3). The value of upper bound $Q_{\mathrm{e}}$ is not estimated in the absence of matter-sector physics of the field theories with the Lifshitz point, so is not the width of the white band.

\subsection{Exact solutions with matters of power-law behavior}

Next let us assume some matter distribution showing the following power-law behavior

$$
\mathcal{L}_{\mathrm{M}}-\frac{\partial \mathcal{L}_{\mathrm{M}}}{\partial f}=\frac{A}{r^{a}}, \quad \mathcal{L}_{\mathrm{M}}+\frac{\partial \mathcal{L}_{\mathrm{M}}}{\partial h}=\frac{B}{r^{b}},
$$

where $A$ and $B$ are undetermined constants. Substituting the configuration (3.12) into the constraint equation (3.2), we obtain $a=b$ and $B=(1-a / 2) A$. With these relations we solve Eq. (3.1) exactly and find the solutions

$$
n(r)= \begin{cases} \pm \sqrt{-\frac{\sigma}{6 \alpha} r^{2}+\frac{f_{0} \xi}{\alpha}+\frac{n_{0}}{r}-\frac{f_{0}^{2}(5 \zeta+14 \eta)}{\alpha r^{2}}-\frac{A r^{2-a}}{2 \alpha(3-a)}}, \quad(a \geq 0, a \neq 3) \\ \pm \sqrt{-\frac{\sigma}{6 \alpha} r^{2}+\frac{f_{0} \xi}{\alpha}+\frac{n_{0}}{r}-\frac{f_{0}^{2}(5 \zeta+14 \eta)}{\alpha r^{2}}-\frac{A \ln r}{2 \alpha r},} \quad(a=3)\end{cases}
$$

Note that the $a=4$ case is nothing but the aforementioned electric field of charge density $q_{\mathrm{e}}$ with $A=-q_{\mathrm{e}}^{2} / 32 \pi^{2}$ and $B=q_{\mathrm{e}}^{2} / 32 \pi^{2}$. A constant matter distribution, the $a=0$ case, affects the cosmological constant. According to power counting, the physics of odd $a$ is unclear as was discussed in the previous work [12]. For the magnetic field of a thin filament of magnetic charge density $q_{\mathrm{m}}$ in IR regime $(z=1)$, we can obtain the same solution (3.5) except for the replacement $q_{\mathrm{e}} \rightarrow q_{\mathrm{m}}$ since the electromagnetic duality of Maxwell theory works. The cases of even $a$ 's maybe interpreted as the magnetically charged thin filament with different anisotropic scalings of $z=a / 2-1$. To be a consistent solution, the field configuration of consideration should 
simultaneously satisfy both the constraint equation (3.2) and the Euler-Lagrange equation from $\mathcal{L}_{\mathrm{M}}$.

An intriguing example of a stringy object showing a power-law behavior in its energy density may be global vortices. The gravitating global vortex in the IR regime of $z=1$ is described by a field theory of a complex scalar field $\phi$ and the Lagrangian density for the $k$ vortices superimposed at the origin is

$$
\mathcal{L}_{\mathrm{M}}=-\frac{1}{2} e^{2 f}|\phi|^{\prime 2}-\frac{k^{2}}{2 r^{2}}|\phi|^{2}-V(|\phi|)
$$

where $V(|\phi|)$ is an arbitrary scalar potential of scalar amplitude $|\phi|$ involving spontaneous symmetry breaking, $|\phi| \stackrel{r \rightarrow \infty}{\rightarrow} v$. It is then natural to ask whether or not the $1 / r^{2}$ term for nonzero vorticity $(k \neq 0)$ leads to a possible $a=2$ solution. In order to be compatible with the metric solution in (2.17) and (2.19), the scalar amplitude should satisfy the constraint equation (3.2) as well as the Euler-Lagrange equation derived from (3.14). A straightforward analysis proves nonexistence of the static global vortex solutions described by (3.14).

\section{Conclusion and Discussion}

In this paper we have considered $(1+3)$-dimensional HL gravity of $z=3$ anisotropic scaling. We employ the version with the projectability condition but impose the detailed balance condition only in the UV fixed point, which results in the Lagrangian containing 8 parameters and the square of the Cotton term as the unique sixth order spatial derivative term. Solving the equations of motion in the Painlevé-Gullstrand type coordinates with axial symmetry, we found exact static stringy solutions which allow various planar geometries: a cone with deficit angle, an excess cone with surplus angle, AdS type black holes, and a charged black hole. The excess cone with surplus angle is a forbidden solution due to violation of the positive energy theorem in GR, but, amazingly enough, it is a generic vacuum solution in HL gravity. The effect of quartic spatial derivative terms is equivalent to the square of the electric field in static solution, of which negative coefficients permit the square of electric field to be negative. In order to obtain such a solution in GR the positive energy theorem is violated. It can also be interpreted as the effect making the square of electric charge negative, which is against repulsive Coulomb force between the same charges in Maxwell theory. The obtained solutions are categorized and tested according to the classification stated in Sec. 1, and the results are summarized in Figs. 2 and 3 and in Table I. 


\begin{tabular}{|c|c|c|c|c|}
\hline Solution & Parameters & Category & Zone & Physics \\
\hline 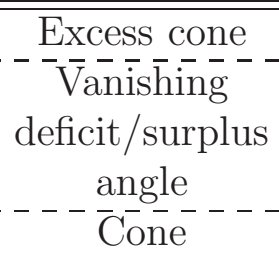 & $\begin{array}{c}f_{0}(1-\xi / \alpha)>1 \\
f_{0}(1-\xi / \alpha)=1 \\
\overline{0}-\overline{f_{0}}(\overline{1}-\bar{\xi} / \bar{\alpha})-\overline{1}--\end{array}$ & $\begin{array}{c}(\mathrm{III}) \\
(\overline{\mathrm{III}})^{-}, \overline{(\overline{\mathrm{II}})}\end{array}$ & Unlikely & $\begin{array}{l}\text { Violation of } \\
\text { the positive energy } \\
\text { theorem in GR }\end{array}$ \\
\hline GR limit & $f_{0}(1-\xi / \alpha)=0$ & (I) & Allowed & Equivalent to GR solution \\
\hline $\begin{array}{c}\text { AdS } \\
\text { black string }\end{array}$ & $f_{0}(1-\xi / \alpha)<0$ & (II) & Disfavored & $\begin{array}{c}\text { Spacetime signature flip } \\
\text { without a negative } \\
\text { cosmological constant }\end{array}$ \\
\hline \multirow{3}{*}{$\begin{array}{l}\text { Charged } \\
\text { (black) } \\
\text { string }\end{array}$} & $5 \zeta+14 \eta<-Q_{\mathrm{e}}^{2} / 64 \pi^{2} f_{0}^{2}$ & (III) & Unlikely & $\begin{array}{l}\text { Attractive Coulomb } \\
\text { force between } \\
\text { the same charges }\end{array}$ \\
\hline & $5 \zeta+14 \eta \approx 0$ & $(\mathrm{I})$ & Allowed & Effectively (almost) neutral \\
\hline & $5 \zeta+14 \eta>Q_{\mathrm{e}}^{2} / 64 \pi^{2} f_{0}^{2}$ & (II) & Disfavored & $\begin{array}{c}\text { Square of effective } \\
\text { excess charge is positive } \\
\text { but too large }\end{array}$ \\
\hline
\end{tabular}

Table 1. Classification of solutions and the categorized zones

The second purpose of this paper was to propose a method to constrain a possible pattern of the RG flows connecting the IR and UV fixed points by utilizing generic classical solutions of HL gravity. Since we cannot directly tackle the quantum regime of HL gravity and cannot compute the RG flows at the moment, this indirect method we propose may provide some hints on viable zones in the space of many parameters. Specifically, in Sec. 1, we suggested the guidelines for selecting classical solutions, 3 categories, and the classification scheme of the parameter regions, 3 zones. However, probing the quantum nature of HL gravity by using classical tools involves ambiguities and jumps. Though we have some generic classical solutions in exact form, some theoretical arbitrariness always exists in categorizing the solutions and selecting the zones. In addition, survival of the corresponding objects and detection of their astrophysical signal in the present universe are endangered by the following possibilities. Above all, their production rate can be low. Even if the objects are materialized in the very early universe, they can be swept away by environmental changes, can be recombined to some different objects by the mutual interactions, can be transformed to fossils in the period of phase transition, and can be diluted in the inflationary era. All of these lead to a rare event rate or feeble signals in astronomical observations. However, if the obtained solutions are generic and the corresponding objects are formed in the epoch of HL gravity, they are likely inherited and their remnants might be yet residing in the present universe.

The above ambiguities of the conclusion on the parameter zones, obtained from a few tests based on the exact classical solutions, are tentative at this moment. Subsequently this indistinct conclusion in the beginning stage even obscures validity of the proposed method itself. However, fortunately enough, many classical solutions, among which some are generic and exact, have 
already been found in HL gravity, and more classical configurations, of which characteristics are drastically different from GR solutions, will be obtained. If these many solutions are applied for attaining a viable pattern of the RG flows and for testing the proposed method, we will have various maps filled with classified zones and their boundaries as given in Figs. 2 and 3 , Then we overlap these maps on each corresponding lower-dimensional scene projected from the multidimensional parameter space. For example, Fig. 2 (Fig. 31) is understood as a (another) map to put on a (a different) 2-dimensional scene projected from our 10-dimensional space of 8 parameters and 2 constants. Once the overlapped data are sufficiently accumulated, the information on every zone becomes manifest, classified by the allowed, disfavored, and unlikely zone that we explained earlier. Then a panoramic view of the whole multidimensional parameter space is also available. This result will suggest a possible pattern of the RG flows, and, if we are lucky enough, will provide some restricted narrow paths for the flows. When we reach a consistent conclusion on the pattern of the RG flows through the tests utilizing classical solutions, it will help to judge whether or not there can exist a viable flow line connecting smoothly the UV fixed point of $z=3$ anisotropic scaling and the IR fixed point of GR. Furthermore, once the aforementioned scenario is proven to be reliable, the panoramic method we propose will be established as an efficient way in studying the RG flows of a premature complicated theory involving too many terms and undetermined coefficients, and finally will play a role of classical predictor for unknown quantum realm.

We conclude this section with a list of further studies. First, we obtained and tested the static solutions with axial symmetry, but it is also worth finding those with spherical symmetry for additional tests [25]. Second, the exact solutions are obtained only for $\lambda=1$. If we can find some exact solutions for arbitrary $\lambda$ despite extreme complication, it will allow a more accurate discussion on the RG flow in the IR limit. Third, our studies focused on the IR regime. To understand quantized HL gravity it is important to figure out physics in the vicinity of the UV fixed point, where higher spatial derivatives satisfying the $z=3$ anisotropic scaling dominate. For the application of the proposed panoramic method, it is intriguing to find generic classical solutions of the nonvanishing Cotton term $C_{i j} \neq 0$. This study in UV regime may also shed light on understanding the phase structure of HL gravity, which is performed only through the comparison to lattice results [16].

\section{Acknowledgments}

The authors would like to thank Cheongho Han, Hang Bae Kim, and Sung-Soo Kim for valuable discussions and comments. This work was supported by the National Research Foundation of Korea(NRF) grant funded by the Korea government (MEST) (No. 2009-0062869) through the Astrophysical Research Center for the Structure and Evolution of the Cosmos (ARCSEC) and by the Korea Research Foundation Grant funded by the Korean Government (KRF-2008-313C00170). 


\section{References}

[1] P. Horava, Phys. Rev. D 79, 084008 (2009) [arXiv:0901.3775 [hep-th]].

[2] P. Horava and C. M. Melby-Thompson, Phys. Rev. D 82, 064027 (2010) arXiv:1007.2410 [hep-th]].

[3] P. Horava, JHEP 0903, 020 (2009) [arXiv:0812.4287 [hep-th]].

[4] P. Horava, Phys. Rev. Lett. 102, 161301 (2009) arXiv:0902.3657 [hep-th]].

[5] R. G. Cai, B. Hu and H. B. Zhang, Phys. Rev. D 80, 041501 (2009) arXiv:0905.0255 [hep-th]]; C. Charmousis, G. Niz, A. Padilla and P. M. Saffin, JHEP 0908, 070 (2009) arXiv:0905.2579 [hep-th]]; D. Blas, O. Pujolas and S. Sibiryakov, JHEP 0910, 029 (2009) arXiv:0906.3046 [hep-th]], Phys. Rev. Lett. 104, 181302 (2010) [arXiv:0909.3525 [hep-th]], Phys. Lett. B 688, 350 (2010) [arXiv:0912.0550 [hep-th]]; C. Bogdanos and E. N. Saridakis, Class. Quant. Grav. 27, 075005 (2010) [arXiv:0907.1636 [hep-th]]; A. Wang and R. Maartens, Phys. Rev. D 81, 024009 (2010) [arXiv:0907.1748 [hep-th]]; K. Koyama and F. Arroja, JHEP 1003, 061 (2010) arXiv:0910.1998 [hep-th]]; A. Papazoglou and T. P. Sotiriou, Phys. Lett. B 685, 197 (2010) arXiv:0911.1299 [hep-th]]; J. O. Gong, S. Koh and M. Sasaki, Phys. Rev. D 81, 084053 (2010) arXiv:1002.1429 [hep-th]].

[6] T. P. Sotiriou, M. Visser and S. Weinfurtner, JHEP 0910, 033 (2009) arXiv:0905.2798 [hep-th]].

[7] M. Li and Y. Pang, JHEP 0908, 015 (2009) [arXiv:0905.2751 [hep-th]]; M. Henneaux, A. Kleinschmidt and G. L. Gomez, Phys. Rev. D 81, 064002 (2010) arXiv:0912.0399 [hepth]]; J. Kluson, JHEP 1007, 038 (2010) [arXiv:1004.3428 [hep-th]].

[8] G. Calcagni, JHEP 0909, 112 (2009) [arXiv:0904.0829 [hep-th]]; E. Kiritsis and G. Kofinas, Nucl. Phys. B 821, 467 (2009) arXiv:0904.1334 [hep-th]]; S. Mukohyama, JCAP 0906, 001 (2009) arXiv:0904.2190 [hep-th]], Phys. Rev. D 80, 064005 (2009) arXiv:0905.3563 [hepth]], JCAP 0909, 005 (2009) [arXiv:0906.5069 [hep-th]]; R. Brandenberger, Phys. Rev. D 80, 043516 (2009) arXiv:0904.2835 [hep-th]].

[9] H. Lu, J. Mei and C. N. Pope, Phys. Rev. Lett. 103, 091301 (2009) arXiv:0904.1595 [hepth]].

[10] A. Kehagias and K. Sfetsos, Phys. Lett. B 678, 123 (2009) arXiv:0905.0477 [hep-th]]; M. i. Park, JHEP 0909, 123 (2009) [arXiv:0905.4480 [hep-th]].

[11] R. G. Cai, L. M. Cao and N. Ohta, Phys. Rev. D 80, 024003 (2009) arXiv:0904.3670 [hepth]]; Y. S. Myung and Y. W. Kim, Eur. Phys. J. C 68, 265 (2010) [arXiv:0905.0179 [hep-th]]; 
A. Ghodsi, arXiv:0905.0836 [hep-th]; R. B. Mann, JHEP 0906, 075 (2009) arXiv:0905.1136 [hep-th]]; A. Ghodsi and E. Hatefi, Phys. Rev. D 81, 044016 (2010) arXiv:0906.1237 [hep-th]]; H. W. Lee, Y. W. Kim and Y. S. Myung, Eur. Phys. J. C 68, 255 (2010) arXiv:0907.3568 [hep-th]]; N. Varghese and V. C. Kuriakose, arXiv:0909.4944 [gr-qc]; E. Kiritsis and G. Kofinas, JHEP 1001, 122 (2010) [arXiv:0910.5487 [hep-th]]; M. R. Setare and D. Momeni, arXiv:0911.1877 [hep-th]; T. Kim and C. O. Lee, arXiv:1002.0784 [hep-th]; E. Gruss, arXiv:1005.1353 [hep-th].

[12] S. S. Kim, T. Kim and Y. Kim, Phys. Rev. D 80, 124002 (2009) [arXiv:0907.3093 [hep-th]]; J. Korean Phys. Soc. 57, 634 (2010).

[13] J. Z. Tang and B. Chen, Phys. Rev. D 81, 043515 (2010) arXiv:0909.4127 [hep-th]]; T. Harada, U. Miyamoto and N. Tsukamoto, arXiv:0911.1187 [gr-qc]; D. Capasso and A. P. Polychronakos, Phys. Rev. D 81, 084009 (2010) arXiv:0911.1535 [hep-th]]; J. Greenwald, A. Papazoglou and A. Wang, Phys. Rev. D 81, 084046 (2010) arXiv:0912.0011 [hepth]].

[14] S. Dutta and E. N. Saridakis, JCAP 1001, 013 (2010) arXiv:0911.1435 [hep-th]], JCAP 1005, 013 (2010) arXiv:1002.3373 [hep-th]]; J. M. Romero, R. Bernal-Jaquez and O. Gonzalez-Gaxiola, Mod. Phys. Lett. A 25, 2501 (2010) [arXiv:1003.0684 [hep-th]].

[15] T. Harko, Z. Kovacs and F. S. N. Lobo, arXiv:0908.2874 [gr-qc]; L. Iorio and M. L. Ruggiero, arXiv:0909.2562 [gr-qc], arXiv:0909.5355 [gr-qc]; V. F. Cardone, N. Radicella, M. L. Ruggiero and M. Capone, arXiv:1003.2144 [astro-ph.CO].

[16] C. Xu and P. Horava, Phys. Rev. D 81, 104033 (2010) [arXiv:1003.0009 [hep-th]].

[17] I. Cho and G. Kang, JHEP 1007, 034 (2010) arXiv:0909.3065 [hep-th]].

[18] M. Banados, C. Teitelboim and J. Zanelli, Phys. Rev. Lett. 69, 1849 (1992) arXiv:hep-th/9204099.

[19] Refer to the web site of High Angular Resolution Astronomy (CHARA) designed for exceptionally high angular resolution: http://www.chara.gsu.edu/CHARA/ It is optical/interferometric array of six telescopes and its individual telescope operates at visible and near IR wavelengths.

[20] A. Vilenkin, Phys. Rev. D 23, 852 (1981). For a review, see A. Vilenkin and E. P. S. Shellard, Cosmic strings and other topological defects, (Cambridge University Press, Cambridge, England, 1984).

[21] K. Kuijken, X. Siemens and T. Vachaspati, Mon. Not. R. Astron. Soc. 384, 161 (2008) arXiv:0707.2971 [astro-ph]]. 
[22] R. G. Cai and A. Wang, Phys. Lett. B 686, 166 (2010) [arXiv:1001.0155 [hep-th]].

[23] G. W. Semenoff, Phys. Rev. Lett. 53, 2449 (1984).

[24] P. Horava, arXiv:0811.2217 [hep-th].

[25] H. B. Kim and Y. Kim, arXiv:1009.1201 [hep-th]. 